\title{
Aetiology and Prevalence of Respiratory Distress in Newborns Delivered at DMCH, Darbhanga, Bihar, India
}

\author{
Kripa Nath Mishra1 ${ }^{1}$ Pankaj Kumar², Prashant Gaurav ${ }^{3}$ \\ 1,2,3 Department of Paediatrics, Darbhanga Medical College and Hospital, Laheriasarai, Darbhanga, Bihar, India.
}

\section{ABSTRACT}

\section{BACKGROUND}

Respiratory distress is one of the commonest disorders and is the commonest cause of morbidity in new-borns. It occurs among $4-7 \%$ of all neonates and is the reason for $30-40 \%$ of admissions in the Neonatal Intensive Care Unit (NICU). Respiratory distress is more common among preterm (30\%) and post term (21\%) than among term neonates (4.2\%). It is defined as presence of the tachypnoea (RR $>60 / \mathrm{min}$ ) with subcostal / intercostals retractions, expiratory grunting / groaning. In addition to the above features, presence of nasal flaring, suprasternal retractions, decrease air entry on chest auscultation also indicate the presence of respiratory distress. Gasping, chocking or stridor (sign of upper airways obstruction), apnoea or poor respiratory effort or bradycardia, poor perfusion and cyanosis are life threatening signs that require prompt intervention. We wanted to study the aetiology and prevalence of respiratory distress in neonates born between February 2019 to January 2020.

\section{METHODS}

This study was done in Darbhanga Medical College \& Hospital, Laheriasarai, Darbhanga, Bihar, India, over a period of one year from February 2019 to January 2020. This is a descriptive study. All live new-borns delivered at Darbhanga Medical College \& Hospital, Laheriasarai, Bihar, India, having signs of respiratory distress and admitted in NICU, Department of Paediatrics, during the study period, were included in the study.

\section{RESULTS}

Respiratory distress was observed in 6120 new-borns $(n=6120)$ delivered over the study duration of 12 months at this hospital. $19.2 \%$ in preterm, $7 \%$ in post-term and $2.2 \%$ in full term. On the whole common cause of respiratory distress was transient tachypnoea of new-born (TTN) which was $35.3 \%$ while hyaline membrane disease (HMD) was $27 \%$ followed by meconium aspiration syndrome (MAS) $18.4 \%$, pneumonia $3.3 \%$, pneumothorax $1.8 \%$ and other congenital anomalies were $14.2 \%$. In both term and late preterm new-borns, TTN was found to be common, while meconium aspiration syndrome was common among term and post term babies.

\section{CONCLUSIONS}

Among the neonatal problems with significant mortality and morbidity, respiratory distress is one of the commonest causes, while TTN was the commonest aetiology. Others are hyaline membrane disease and meconium aspiration syndrome.

\section{KEY WORDS}

New-Born, Respiratory Distress, Transient Tachypnoea of New-Born (TTN), Meconium Aspiration Syndrome (MAS), Hyaline Membrane Disease (Respiratory Distress Syndrome)
Corresponding Author: Dr. Pankaj Kumar, Junior Resident, Department of Paediatrics, DMCH, Laheriasarai, Darbhanga, Bihar-846003, India, E-mail: pankajskmch08@gmail.com

DOI: $10.14260 / j e m d s / 2020 / 802$

How to Cite This Article:

Mishra KN, Kumar P, Gaurav P. Aetiology and prevalence of respiratory distress in newborns delivered at DMCH, Darbhanga, Bihar, India. J Evolution Med Dent Sci 2020;9(48):3655-3659, $10.14260 / \mathrm{jemds} / 2020 / 802$ DOI:

Submission 06-05-2020,

Peer Review 15-10-2020,

Acceptance 21-10-2020,

Published 30-11-2020.

Copyright (C) 2020 Kripa Nath Mishra et al. This is an open access article distributed under Creative Commons Attribution License [Attribution 4.0 International (CC BY 4.0)] 


\section{BACKGROUND}

Respiratory distress is one of the commonest disorders of newborn. It occurs among $4-7 \%$ of all neonates ${ }^{1,2}$ and is the reason for $30-40 \%$ of admissions in the Neonatal Intensive Care Unit (NICU). Respiratory distress is more common among preterm (30\%) and post-term (21\%) than among term neonates $(4.2 \%) .^{2}$ It is defined as presence of the tachypnoea (RR > $60 / \mathrm{min}$ ) with subcostal / intercostals retractions, expiratory grunting / groaning. In addition to the above features, presence of nasal flaring, suprasternal retractions, decrease air entry on auscultation of the chest also indicates the presence of respiratory distress. Gasping, chocking or stridor (sign of upper airways obstruction), apnoea or poor respiratory effort or bradycardia, poor perfusion and cyanosis are life threatening signs that require prompt intervention. ${ }^{3}$

Severity of respiratory distress in preterm babies is assessed by Silverman Anderson score ${ }^{4}$ and in term babies it is assessed by Downe's score ${ }^{5}$. Respiratory distress in neonates can be due to wide variety of conditions including transient tachypnoea of the new-born (TTN), hyaline membrane disease (HMD), meconium aspiration syndrome (MAS), pneumonia, persistent pulmonary hypertension of new-born, and non-respiratory disorders like cardiac, renal, gastrointestinal, neurological diseases and congenital anomalies. The frequency of a given above condition depends on various factors of which gestation is an important one. Respiratory distress syndrome is the most common cause in preterm neonates while Transient tachypnoea of newborn (TTN) is the predominant cause in late preterm and term neonates. Respiratory distress syndrome (RDS), earlier known as Hyaline membrane disease is caused by insufficient pulmonary surfactant in alveoli that occurs mainly in premature newborn, its frequency is inversely linked to gestational age and birth weight. Its occurs in $60-80 \%$ of newborn less than 28 wk. gestational age (GA) in $15-30 \%$ of those between 32 and $36 \mathrm{wk}$. GA, and infrequently in those more than 37 wk. GA. The risk of developing Respiratory Distress Syndrome increased in diabetic mother, new-born delivered by precipitous labour, history of asphyxia, exposure to cold, and a bad obstetrical history of previously affected new-born. The possibility of RDS is low in pregnancies with chronic or pregnancy associated hypertension, maternal heroin use, prolong rupture of membranes, and antenatal corticosteroids prophylaxis. Deficiency of Surfactant is the primary cause of RDS. In absence of pulmonary surfactant, significantly increase alveolar surface tension leads to atelectasis. Sufficient amounts of pulmonary surfactant are present generally after 35 wk. of gestation. Clinical sign of Respiratory Distress Syndrome usually appear just after birth, although larger proportion of premature infants may go unrecognised for several hours, until they presents with rapid and shallow breathing become more obvious. A tachypnoea of late onset should suggest other conditions. Due to intrapartum asphyxia or initial severe respiratory distress (mainly neonates having birth weight less than $1000 \mathrm{~g}$ ) may require resuscitation at birth. The untreated RDS can lead to progressive worsening of cyanosis and dyspnoea. Inadequate pulmonary $\mathrm{O}_{2}-\mathrm{CO}_{2}$ gas exchange is the basic pathology required for the treatment of
RDS. Basic supportive care (thermoregulatory, circulatory, fluid, electrolyte, and respiratory) is essential. The watchful and repeated evaluation of heart rate and respiratory rate, $\mathrm{SaO}_{2}, \mathrm{PaO}_{2}, \mathrm{PaCO}_{2}$, $\mathrm{pH}$, electrolytes, glucose, haematocrit, blood pressure and temperature are necessary. Regular evaluation of $\mathrm{PaO}_{2}, \mathrm{PaCO}_{2}$ and $\mathrm{pH}$ is an essential element of the management and is to provide support care; in assisted ventilation, such evaluation is being essential. The basic pathophysiology of RDS is surfactant deficiency. Instant effects of pulmonary surfactant replacement therapy include enhanced alveolar-arterial oxygen gradient, reduced ventilator support, increased pulmonary compliance and improved chest radiograph appearance.

Transient tachypnoea of the newborn (TTN) is a clinical syndrome of self-limited tachypnoea associated with delayed clearance of fetal lung fluid. Twin gestation, maternal asthma, late prematurity, precipitous delivery, gestational diabetes, caesarean delivery without labour are common associated risk factors. Clearance of fetal lung fluid occurs through increase expression of epithelial sodium channels $(\mathrm{ENaC})$ and sodiumpotassium adenosine triphosphate $(\mathrm{Na}+, \mathrm{K}+-$ ATPase $)$ that drive active sodium (and thereby fluid) reabsorption. TTN is believed to result from ineffective expression or activity of $\mathrm{ENaC}$ and $\mathrm{Na}+, \mathrm{K}+-$ ATPase, which slow absorption of fetal lung fluid and results in decrease pulmonary compliance and impeded gas exchange. Treatment of TTN is mainly supportive. Inhaled beta-2 agonist such as albuterol (salbutamol) increase expression and activation of ENaC and $\mathrm{Na}+, \mathrm{K}+-$ ATPase and facilitate fluid clearance. Increased risk for developing respiratory distress in term newborns is associated with newborns delivered by caesarean section as compared with those born by vaginal delivery ${ }^{6}$. Increased rate of caesarean section leading to increased prevalence of newborns admitted to hospital with complain of respiratory distress. $^{7}$

In term and post-term newborns Meconium aspiration syndrome (MAS) is one of the most common aetiology of respiratory distress. The overall frequency lies between $5 \%$ and $25 \%$ (median $14 \%$ ) of meconium stained amniotic fluid (MSAF). MAS occurs in about $10 \%$ of infants born through MSAF. Tachypnoea, chest retractions, grunting and cyanosis are common clinical features due to obstruction of small airway, while pneumomediastinum, pneumothorax or both are due to partial obstruction of airways. The prominent sign of MAS is chest overdistension. Usually these signs were improved within 72 hours, the risk of mortality is increased, when symptoms become severe leading to require assisted ventilation. On chest radiography, patchy infiltrations, coarse streaking in both lung fields, increased antero-posterior diameter of chest and diaphragm flattening. The rapid recognition of fetal distress and beginning of timely delivery in the presence of late fetal heart deceleration or poor beat-tobeat FHR variability leading to decreased risk of meconium aspiration. The standard management of MAS incudes supportive care and symptomatic treatment for respiratory distress. The oxygenation to reduce mean airway pressure and risk of pneumothorax should be weighted. In new-borns with MAS and hypoxic respiratory failure requiring mechanical ventilation administration of exogenous surfactant and / or iNO, decreases the need for extracorporeal membrane 
oxygenation in new-borns affected most severely, who show no response to normal standard therapy. Persistent pulmonary hypertension is the complication severe meconium aspiration. HFV or ECMO may be beneficiary to patients with MAS which are refractory to conventional mechanical ventilation. The mortality rate is higher in meconium stained infants in comparison to unstained infants. With the advancement in obstetric and neonatal care, there is decrease in neonatal deaths related to MAS in recent decades.

\section{METHODS}

In this descriptive study, total 6120 live new-born were selected by purposive sampling technique. Selection within the strata is done for convenience. All these live new-born babies delivered at Darbhanga Medical College \& Hospital, Laheriasarai, Darbhanga, Bihar during the study period from February 2019 to January 2020 were observed for respiratory distress and the objective was to establish or determine the prevalence and aetiology of respiratory distress in inborn new-born babies. For this study informed consent from parents / guardian were taken. Approval of the institutional ethical committee was taken prior to conduct of this study.

\section{Inclusion Criteria}

- All inborn new-born babies admitted in NICU include term, preterm, post term up to 28 days.

- Babies delivered by Caesarean section or vaginal deliveries.

\section{Exclusion Criteria}

- Babies more than 28 days

- Out born babies

- Babies with congenital malformations like meningocele, meningomyelocele, encephalocele and anencephaly.

\section{Investigations}

CBC, CRP, blood glucose, blood culture, electrolytes, cranial ultrasound, chest x-rays, pulse oximetry, 2DEchocardiography.

Any new-born babies showing any two or more clinical signs was suspected to have respiratory distress having respiratory rate of $\geq 60 /$ minute, subcostal / intercostal recessions and expiratory grunt / groaning in addition to these features that is presence of nasal flaring, suprasternal retractions, decrease air entry on auscultation of the chest.

Data was collected for all newborn babies after the initial evaluation and cardio pulmonary management, a detailed history was collected. General information, socioeconomic status, history of mother and her current and previous antenatal histories were taken which provided indispensable statistics. Intra-partum particulars with peculiar reference to the fetal well-being, quantity and quality of liquor, duration of the rupture of membrane, drugs given to the mother were recorded. Sex, birth weight, Apgar score, resuscitation particulars, and on the basis of last date of menstrual period gestational age was calculated and clinical examination findings expressive of respiratory difficulties were also recorded. Time of onset and the severity of the respiratory distress was documented and the severity was assessed by using Downe's score and Silverman Anderson score. All these data was collected for all new-born include in study with respiratory distress. All new-borns with respiratory distress were managed in NICU. Investigations related to clinical conditions were obtained in all cases. Sepsis screen and blood culture were indicated when infection was suspected, and echocardiography were done whenever indicated (to ruled out congenital heart disease and to evaluate Persistent pulmonary hypertension). All new-born babies with respiratory distress received supportive and standard care with recording of vital signs and oxygenation saturation, respiratory support in severe condition as respiratory failure.

\section{Statistical Analysis}

Data was collected for all new born include in our study. Descriptive statistics were used for analysis of results.

\section{RESULTS}

The overall number of live inborn births was 6,120 through 1 year of study period. Out of which 4,284 were spontaneous vaginal deliveries (SVD) while 1836 were delivered by caesarean section. Out of these newborns 269 had evolved respiratory distress. The sex ratio between male and female newborn babies was 1:1. 144 newborns were premature, 115 newborns were full term and 10 newborns were post term. The mean age of newly born babies were 210 minutes. In my present study overall respiratory distress prevalence was 4.4 $\%$. Out of which prevalence of respiratory distress were 19.2 $\%, 2.2 \%$ and $7 \%$ among preterm, term and post-term respectively. The most important etiology of RD are shown in (Table 1). In this study the commonest cause of respiratory distress was found to be transient tachypnea of newborn (TTN) $35.3 \%$ which was followed by hyaline membrane disease (HMD) $27 \%$, and $18.4 \%$ have meconium aspiration syndrome (MAS), $3.3 \%$ and $1.8 \%$ have pneumonia and pneumothorax respectively and $14.2 \%$ have other congenital anomalies including CHD (Congenital Heart Disease), CDH (Congenital Diaphragmatic Hernia), choanal atresia, TEF (Tracheo-Esophageal Fistula) \& pierre robin syndrome.

\section{DISCUSSION}

In this present study overall respiratory distress prevalence was $4.4 \%$. While other previous studies done in developed countries have prevalence ranging from $3 \%$ to $7 \%{ }^{8,9-14}$ These results from our study were equivalent with other results from different studies done in developed countries of world (Alok Kumar et $\mathrm{al}^{2}$ Malhotra et $\mathrm{al}^{15}$ ).

The factors which influenced the overall distribution of respiratory distress in new-born are geographic distribution, ethnic factors and standard protocol of care in NICU 
availability These results from our study were equivalent with other results from different studies done in developed countries of world In this study the commonest cause of respiratory distress was found to be transient tachypnoea of newborn (TTN) $35.3 \%$ which was followed by hyaline membrane disease (HMD) $27 \%$, and $18.4 \%$ have meconium aspiration syndrome (MAS), $3.3 \%$ and $1.8 \%$ have pneumonia and pneumothorax respectively and $14.2 \%$ have other congenital anomalies including CHD (Congenital Heart Disease), CDH (Congenital Diaphragmatic Hernia), choanal atresia, TEF (Tracheo-Oesophageal Fistula) \& Pierre Robin syndrome.

There is similarities in the pattern of respiratory distress between our studies and the studies done from different developed countries. ${ }^{5,16}$ In preterm infants the most common cause of Respiratory distress syndrome is Hyaline Membrane Disease (HMD), now known as Respiratory distress syndrome of newborn, which is caused by insufficient pulmonary surfactant in the alveoli. In this study among pre-term newborns the prevalence of respiratory distress was $19.2 \%$ that was comparable to different studies done in other developed countries which range from $28 \%$ to $53 \%$. In another studies whose result was quite comparable to our study, done in Aga Khan university Hospital Karachi Pakistan, result ranges from $12.8 \%$ to $45 \% .{ }^{17}$ In another study whose result ranges from $10 \%$ to $16 \%$ closely correlates with our studies in term and post-term newborns, the prevalence of Meconium aspiration syndrome was found in $18.4 \%$ cases.

\begin{tabular}{|ccc|}
\hline Primary Diagnosis & Frequency & Percentage \\
\hline Transient Tachypnoea of Newborn & 95 & $35.3 \%$ \\
Respiratory Distress Syndrome & 73 & $27 \%$ \\
Meconium Aspiration Syndrome & 49 & $18.4 \%$ \\
Others (Congenital Heart Disease / Choanal Atresia / & & \\
Tracheo-Oesophageal Fistula / Congenital & 38 & $14.2 \%$ \\
Diaphragmatic Hernia / Pierre Robin Syndrome) & & \\
Pneumonia & 09 & $3.3 \%$ \\
Pneumothorax & 05 & $1.8 \%$ \\
\hline Table 1 Causes of Respiratory Distress in New-Born $(\boldsymbol{n}=\mathbf{2 6 9})$ \\
\hline
\end{tabular}

\section{CONCLUSIONS}

Respiratory distress in newborn is one of the commonest causes of admission in NICU, with significant morbidity and mortality. TTN is found in the majority of the cases in term babies whereas hyaline membrane disease is common in preterm babies. TTN is a self-limiting condition which needs only supportive treatment and generally resolves after minimal therapeutic intervention. Giving antenatal corticosteroids to the mother before 37 weeks of gestation can significantly reduce prevalence of HMD. With improvement in obstetrical care, neonatal management, symptomatic care and standard treatment of respiratory distress reduces the cases because of MAS. Pneumonia, pneumo-thorax, septicemia, CNS causes, congenital heart disease and other congenital malformations are the less common causes of respiratory distress, which are managed by disease-oriented treatment. Hence, to reduce morbidity and mortality due to respiratory distress, early diagnosis and prompt treatment are necessary.
Data sharing statement provided by the authors is available with the full text of this article at jemds.com.

Financial or other competing interests: None.

Disclosure forms provided by the authors are available with the full text of this article at jemds.com.

\section{REFERENCES}

[1] Misra PK. Respiratory distress in newborn. A prospective study. Indian Pediatr 1987;24(1):77-80.

[2] Kumar A, Bhat BV. Epidemiology of respiratory distress of newborns. Indian J Pediatr 1996;63(1):93-8.

[3] Aly H. Respiratory disorder in the newborn: identification and diagonosis. Pediatr Rev 2004;25(6):201-8.

[4] Silverman WA, Anderson DH. A controlled clinical trial of effects of water mist on obstructive respiratory signs, death rate and necropsy findings among premature infants. Pediatrics 1956;17(1):1-10.

[5] Downes JJ, Vidyasagar D, Boggs TR, et al. Respiratory distress syndrome of newborn infants. I. New clinical scoring system (RDS score) with acid--base and blood gas correlations. Clin Pediatr (Phila) 1970;9(6):325-31.

[6] Kolas T, Saugstad OD, Daltveit AK, et al. Planned caesarean versus planned vaginal delivery at term: comparision of newborn infant outcomes. Am J Obstet Gynecol 2006;195(6):1538-43.

[7] Ersch J, Roth-Kleiner M, Baeckert $P$, et al. Increasing incidence of respiratory distress in neonates. Acta Paediatr 2007;96(11):1577-81.

[8] Hermansen CL, Lorah KN. Respiratory distress in the newborn. Am Fam Physcian 2007;76(7):987-94.

[9] Bonafe L, Rubaltelli FF. The incidence of acute neonatal respiratory disorders in Padova county: an epidemiological survey. Acta Paediatr 1996;85(10):123640.

[10] Nielsen TF, Hokegard KH. The incidence of acute neonatal respiratory disorders in relation to mode of delivery. Acta Obstet Gynecol Scand 1984;63:109-14.

[11] Navaei F, Aliabadi B, Moghtaderi M, et al. Predisposing factors, incidence and mortality of pneumothorax in a neonatal intensive care unit in Isfahan, Iran. Zhongguo Dang Dai Er Ke Za Zhi 2010;12(6):417-20.

[12] Le Ray C, Boithias C, Castaigne-Meary V, et al. Caesarean before labour between 34 and 37 weeks: what are the risk factors of severe neonatal respiratory distress? Eur J Obstet Gynecol Reprod Biol 2006;127(1):56-60.

[13] Yoder BA, Gordon MC, Barth WHJ. Late-preterm birth: does the changing obstetric paradigm alter the epidemiology of respiratory complications? Obstet Gynecol 2008;111(4):814-22.

[14] Fedakar A, Aydogdu C. Clinical features of neonates treated in the intensive care unit for respiratory distress. Turk J Pediatr 2011;53(2):173-9.

[15] Malhotra AK, Nagpal R, Gupta RK, et al. Respiratory distress in newborn: treated with ventilation in a level II nursery. Indian Pediatr 1995;32(2):207-11.

[16] Kumar A, Bhatnagar V. Respiratory distress in neonates. Indian J Pediatr 2005;72(5):425-8.

[17] Ventolini G, Neiger R, Mathews L, et al. Incidence of respiratory disorders in neonates born between 34 and 36 weeks of gestation following exposure to antenatal 
corticosteroids between 24 and 34 weeks of gestation.

Am J Perinatol 2008;25(2):79-83. 